\title{
WEAK FORM OF HOLZAPFEL'S CONJECTURE
}

\author{
AZNIV KASPARIAN AND BORIS KOTZEV
}

Communicated by Vasil V. Tsanov

\begin{abstract}
Let $\mathbb{B} \subset \mathbb{C}^{2}$ be the unit ball and $\Gamma$ be a lattice of $\operatorname{SU}(2,1)$. Bearing in mind that all compact Riemann surfaces are discrete quotients of the unit disc $\Delta \subset \mathbb{C}$, Holzapfel conjectures that the discrete ball quotients $\mathbb{B} / \Gamma$ and their compactifications are widely spread among the smooth projective surfaces. There are known ball quotients $\mathbb{B} / \Gamma$ of general type, as well as rational, abelian, $\mathrm{K} 3$ and elliptic ones. The present note constructs three non-compact ball quotients, which are birational, respectively, to a hyperelliptic, Enriques or a ruled surface with an elliptic base. As a result, we establish that the ball quotient surfaces have representatives in any of the eight Enriques classification classes of smooth projective surfaces.
\end{abstract}

\section{Introduction}

In his monograph [4] Rolf-Peter Holzapfel states as a working hypothesis or a philosophy that "... up to birational equivalence and compactifications, all complex algebraic surfaces are ball quotients." By a complex algebraic surface is meant a smooth projective surface over $\mathbb{C}$. These have smooth minimal models, which are classified by Enriques in eight types - rational, ruled of genus $\geq 1$, abelian, hyperelliptic, K3, Enriques, elliptic and of general type. The compact torsion free ball quotients $\mathbb{B} / \Gamma$ are smooth minimal surfaces of general type. Ishida [10], Keum [11,12] and Dzambic [1] obtain elliptic surfaces, which are minimal resolutions of the isolated cyclic quotient singularities of compact ball quotients. Hirzebruch [2] and then Holzapfel [3], [7], [9] have constructed torsion free ball quotient compactifications with abelian minimal models. In [9] Holzapfel provides a ball quotient compactification, which is birational to the Kummer surface of an abelian surface, i.e., to a smooth minimal K3 surface. Rational ball quotient surfaces are explicitly recognized and studied in [6], [8]. The present work constructs smooth ball quotients with a hyperelliptic or, respectively, a ruled model with an elliptic base. It provides also a ball quotient with one double point, which is birational to an Enriques surface. All of them are finite Galois quotients of a 
non-compact torsion free $\mathbb{B} / \Gamma_{-1}^{(6,8)}$, constructed by Holzapfel in [9] and having abelian minimal model of the toroidal compactification. As a result, we establish the following

Theorem 1 (Weak Form of Holzapfel's Conjecture) . Any of the eight Enriques classification classes of complex projective surfaces contains a ball quotient surface.

\section{Ball Quotient Compactifications with Abelian Minimal Models}

Let us recall that the complex two-ball

$$
\mathbb{B}=\left\{\left(z_{1}, z_{2}\right) \in \mathbb{C}^{2} ;\left|z_{1}\right|^{2}+\left|z_{2}\right|^{2}<1\right\}=\mathrm{SU}(2,1) / \mathrm{S}(\mathrm{U}(2) \times \mathrm{U}(1))
$$

is an irreducible non-compact Hermitian symmetric space. The discrete biholomorphism groups $\Gamma \subset \mathrm{SU}(2,1)$ of $\mathbb{B}$, whose quotients $\mathbb{B} / \Gamma$ have finite $\mathrm{SU}(2,1)$ invariant measure are called ball lattices. The present section studies the image $T$ of the toroidal compactifying divisor $T^{\prime}=(\mathbb{B} / \Gamma)^{\prime} \backslash(\mathbb{B} / \Gamma)$ on the minimal model $A$ of $(\mathbb{B} / \Gamma)^{\prime}$, whenever $A$ is an abelian surface. It establishes that for any subgroup $H \subseteq \operatorname{Aut}(A, T)$ there is a ball quotient $\mathbb{B} / \Gamma_{H}$, birational to $A / H$.

Lemma 2. If a ball quotient $\mathbb{B} / \Gamma$ is birational to an abelian surface $A$ then $\mathbb{B} / \Gamma$ is smooth and non-compact.

Proof: Assume that $\mathbb{B} / \Gamma$ is singular. For a compact $\mathbb{B} / \Gamma$ set $U=\mathbb{B} / \Gamma$. If $\mathbb{B} / \Gamma$ is non-compact, let $U=(\mathbb{B} / \Gamma)^{\prime}$ be the toroidal compactification of $\mathbb{B} / \Gamma$. In either case $U$ is a compact surface with isolated cyclic quotient singularities. Consider the minimal resolution $\varphi: Y \rightarrow U$ of $p_{i} \in U^{\text {sing }}$ by Hirzebruch-Jung strings $E_{i}=\sum_{t=1}^{\nu_{i}} E_{i}^{t}$. The irreducible components $E_{i}^{t}$ of $E_{i}$ are smooth rational curves of self-intersection $\left(E_{i}^{t}\right)^{2} \leq-2$. The birational morphism $Y \rightarrow \rightarrow A$ transforms $E_{i}^{t}$ onto rational curves on $A$. It suffices to observe that an abelian surface $A$ does not support rational curves $C$, in order to conclude that $\mathbb{B} / \Gamma$ is smooth. The compact smooth ball quotients are known to be of general type, so that $\mathbb{B} / \Gamma$ is to be non-compact.

Assume that there is a rational curve $C \subset A$. Its desingularization $f: \widetilde{C} \rightarrow C$ can be viewed as a holomorphic map $F: \widetilde{C} \rightarrow A$. Homotopy lifting property applies to $F$ and provides a holomorphic immersion $\widetilde{F}: \widetilde{C} \rightarrow \widetilde{A}=\mathbb{C}^{2}$ in the universal cover $\widetilde{A}$ of $A$, due to simply connectedness of the smooth rational curve 
$\widetilde{C}$. Its image $\widetilde{F}(\widetilde{C})$ is a compact complex-analytic subvariety of $\mathbb{C}^{2}$, which maps to compact complex-analytic subvarieties $\operatorname{pr}_{i}(\widetilde{F}(\widetilde{C})) \subset \mathbb{C}$ by the canonical projections $\operatorname{pr}_{i}: \mathbb{C}^{2} \rightarrow \mathbb{C}, 1 \leq i \leq 2$. Thus, $\operatorname{pr}_{i}(\widetilde{F}(\widetilde{C}))$ and, therefore, $\widetilde{F}(\widetilde{C})$ are finite. The contradiction justifies the non-existence of rational curves on $A$.

The next lemma lists some immediate properties of the image $T$ of the toroidal compactifying divisor $T^{\prime}$ of $A^{\prime}=(\mathbb{B} / \Gamma)^{\prime}$ on its abelian minimal model $A$.

Lemma 3. Let $A^{\prime}=(\mathbb{B} / \Gamma)^{\prime}$ be a smooth toroidal ball quotient compactification, $\xi: A^{\prime} \rightarrow A$ be the blow-down of the $(-1)$-curves $L=\sum_{j=1}^{s} L_{j}$ on $A^{\prime}$ to an abelian surface $A$ and $T_{i}^{\prime}, 1 \leq i \leq h$ be the disjoint smooth elliptic irreducible components of the toroidal compactifying divisor $T^{\prime}=(\mathbb{B} / \Gamma)^{\prime} \backslash(\mathbb{B} / \Gamma)$. Then

i) $T_{i}=\xi\left(T_{i}^{\prime}\right)$ are smooth irreducible elliptic curves on $A$

ii) $T^{\text {sing }}=\sum_{1 \leq i<j \leq h} T_{i} \cap T_{j}=\xi(L)$

iii) $T_{i} \cap T^{\operatorname{sing}} \neq \emptyset$ and the restrictions $\xi: T_{i}^{\prime} \rightarrow T_{i}$ are bijective for all $1 \leq i \leq h$.

Proof: i) According to the birational invariance of the genus, the curves $T_{i}=$ $\xi\left(T_{i}^{\prime}\right)$ have smooth elliptic desingularizations. It suffices to show that any curve $C \subset A$ of genus one is smooth. If $C$ is singular then its desingularization $\widetilde{C}$ is a smooth elliptic curve. Therefore, the composition $\widetilde{C} \rightarrow C \hookrightarrow A$ of the desingularization map with the identical inclusion of $C$ is a morphism of abelian varieties. In particular, it is unramified, which is not the case for $\widetilde{C} \rightarrow C$. Therefore any curve $C \subset A$ of genus one is smooth.

ii) The inclusion $T^{\text {sing }} \subseteq \sum_{1 \leq i<j \leq h} T_{i} \cap T_{j}$ follows from i). For the opposite inclusion, note that $\left.\xi\right|_{A^{\prime} \backslash L}=\operatorname{Id}_{\left(A^{\prime} \backslash L\right)}: A^{\prime} \backslash L \rightarrow A \backslash \xi(L)$ guarantees $T_{i}=$ $\xi\left(T_{i}^{\prime}\right) \neq \xi\left(T_{j}^{\prime}\right)=T_{j}$ and different elliptic curves on an abelian surface intersect transversally at any of their intersection points. Thus, $T^{\operatorname{sing}}=\sum_{1 \leq i<j \leq h} T_{i} \cap T_{j}$. The disjointness of $T_{i}^{\prime}$ yields $\sum_{1 \leq i<j \leq h} T_{i} \cap T_{j} \subseteq \xi(L)$. Conversely, the Kobayashi hyperbolicity of $\mathbb{B} / \Gamma$ requires card $\left(L_{j} \cap T^{\prime}\right) \geq 2$ for all $1 \leq j \leq s$. However, $\operatorname{card}\left(L_{j} \cap T_{i}^{\prime}\right) \leq 1$ by the smoothness of $T_{i}=\xi\left(T_{i}^{\prime}\right)$, so that there exist at least two $T_{i}^{\prime} \neq T_{k}^{\prime}$ with $\operatorname{card}\left(L_{j} \cap T_{i}^{\prime}\right)=\operatorname{card}\left(L_{j} \cap T_{k}^{\prime}\right)=1$. In other words, the point 
$\xi\left(L_{j}\right) \in T_{i} \cap T_{k}$. That verifies the inclusion $\xi(L) \subseteq \sum_{1 \leq i<j \leq h} T_{i} \cap T_{j}$, whereas the coincidence $\xi(L)=\sum_{1 \leq i<j \leq h} T_{i} \cap T_{j}$.

iii) If $T_{i} \cap \xi(L)=\emptyset$ then the intersection numbers $\left(T_{i}^{\prime}\right)^{2}=T_{i}^{2}$ coincide. By the Adjunction Formula

$$
0=-e\left(T_{i}\right)=T_{i}^{2}+K_{A} \cdot T_{i}=T_{i}^{2}+\mathcal{O}_{A} \cdot T_{i}=T_{i}^{2}
$$

so that $\left(T_{i}^{\prime}\right)^{2}=0$. That contradicts the contractibility of $T_{i}^{\prime}$ to the corresponding cusp of $\mathbb{B} / \Gamma$ and justifies $T_{i} \cap T^{\operatorname{sing}} \neq \emptyset$ for all $1 \leq i \leq h$.

Note that $\left.\xi\right|_{T_{i}^{\prime} \backslash L}=\left.\operatorname{Id}\right|_{T_{i}^{\prime} \backslash L}: T_{i}^{\prime} \backslash L \rightarrow T_{i} \backslash \xi(L)$ is bijective. In order to define $\xi^{-1}: T_{i} \cap \xi(L) \rightarrow T_{i}^{\prime} \cap L$, let us recall that for any $p \in \xi(L)$ the smooth rational curve $\xi^{-1}(p)$ has card $\left(\xi^{-1}(p) \cap T_{i}^{\prime}\right) \leq 1$. More precisely, $\operatorname{card}\left(\xi^{-1}(p) \cap T_{i}^{\prime}\right)=1$ if and only if $p \in T_{i}$, so that for any $p \in T_{i} \cap \xi(L)$ there is a unique point $\{q(p)\}=T_{i}^{\prime} \cap \xi^{-1}(p)$. That provides a regular morphism $\xi^{-1}(p)=q(p)$ for all $p \in T_{i} \cap \xi(L)$.

According to Lemma 3, the image $T=\xi\left(T^{\prime}\right)$ of the toroidal compactifying divisor $T^{\prime}=(\mathbb{B} / \Gamma)^{\prime} \backslash(\mathbb{B} / \Gamma)$ under the blow-down $\xi:(\mathbb{B} / \Gamma)^{\prime} \rightarrow A$ of the $(-1)$-curves is a multi-elliptic divisor, i.e., $T=\sum_{i=1}^{h} T_{i}$ has smooth elliptic irreducible components $T_{i}$, which intersect transversally. Note also that $(A, T)$ determines uniquely $(\mathbb{B} / \Gamma)^{\prime}$ as the blow-up of $A$ at $T^{\text {sing. }}$.

Definition 4. A pair $(A, T)$ of an abelian surface $A$ and a divisor $T \subset A$ is an abelian ball quotient model if there exists a torsion free toroidal ball quotient compactification $(\mathbb{B} / \Gamma)^{\prime}$, such that the blow-down $\xi:(\mathbb{B} / \Gamma)^{\prime} \rightarrow A$ of the $(-1)$ curves on $(\mathbb{B} / \Gamma)^{\prime}$ maps the pair $\left((\mathbb{B} / \Gamma)^{\prime}, T^{\prime}=(\mathbb{B} / \Gamma)^{\prime} \backslash(\mathbb{B} / \Gamma)\right)$ onto $(A, T)$.

The next lemma explains the construction of non-compact ball quotients, which are finite Galois quotients of torsion free non-compact $\mathbb{B} / \Gamma$, birational to abelian surfaces.

Lemma 5. Let $A^{\prime}=(\mathbb{B} / \Gamma)^{\prime}=(\mathbb{B} / \Gamma) \cup T^{\prime}$ be a torsion free ball quotient compactification by a toroidal divisor $T^{\prime}, \xi: A^{\prime} \rightarrow A$ be the blow-down of the (-1)curves on $A^{\prime}$ to the abelian minimal model $A$ and $T=\xi\left(T^{\prime}\right)$. Then

i) $\operatorname{Aut}(A, T)=\operatorname{Aut}\left(A^{\prime}, T^{\prime}\right)$ is a finite group 
ii) any subgroup $H \subseteq \operatorname{Aut}(A, T)$ lifts to a ball lattice $\Gamma_{H}$, such that $\Gamma$ is a normal subgroup of $\Gamma_{H}$ with quotient group $\Gamma_{H} / \Gamma=H$ and $\mathbb{B} / \Gamma_{H}$ is a non-compact ball quotient, birational to $X=A / H$.

Moreover, if $X=A / H$ is a smooth surface then $\mathbb{B} / \Gamma_{H}$ is a smooth ball quotient.

Proof: i) If $G=\operatorname{Aut}(A, T)$, then Lemma 3 ii) implies the $G$-invariance of $\xi(L)$. By the means of an arbitrary automorphism of the smooth projective line $\mathbb{P}^{1}$, one extends the $G$-action to $L$ and, therefore, to

$$
A^{\prime}=\left(A^{\prime} \backslash L\right) \cup L=(A \backslash \xi(L)) \cup L .
$$

The $G$-invariance of $T^{\prime}=\sum_{i=1}^{h} T_{i}^{\prime}$ follows from Lemma 3 iii). That justifies the inclusion $G \subseteq \operatorname{Aut}\left(A^{\prime}, T^{\prime}\right)$. For the opposite inclusion, note that the union $L$ of the $(-1)$-curves is invariant under an arbitrary automorphism of $A^{\prime}$. As a result, there arises a $G$-action on $\xi(L)$ and $A=(A \backslash \xi(L)) \cup \xi(L)=\left(A^{\prime} \backslash L\right) \cup \xi(L)$. The multi-elliptic divisor $T=\sum_{i=1}^{h} T_{i}$ is $G$-invariant according to Lemma 3 iii). Consequently, $\operatorname{Aut}\left(A^{\prime}, T^{\prime}\right) \subseteq G$, whereas $G=\operatorname{Aut}\left(A^{\prime}, T^{\prime}\right)$.

In order to show that $G$ is finite, let us consider the natural representation

$$
\varphi: G \longrightarrow \operatorname{Sym}\left(T_{1}, \ldots, T_{h}\right) \simeq \operatorname{Sym}_{h}
$$

in the permutation group of the irreducible components $T_{i}$ of $T$. It suffices to prove that the kernel $\operatorname{ker} \varphi$ is finite, in order to assert that $G$ is finite. For any $g=\tau_{p} g_{o} \in \operatorname{ker} \varphi \subset \operatorname{Aut}(A)$ with linear part $g_{o} \in \mathrm{GL}_{2}(\mathbb{C})$ and translation part $\tau_{p}, p \in A$, we show that $g_{o}$ and $\tau_{p}$ take finitely many values. Note that the identical inclusions $T_{i} \subset A$ are morphisms of abelian varieties. Thus, for any choice of an origin $\check{o}_{A} \in T_{i}$ there is a $\mathbb{C}$-linear embedding $\mathcal{E}_{i}: \widetilde{T}_{i}=\mathbb{C} \hookrightarrow \mathbb{C}^{2}=\widetilde{A}$ of the corresponding universal covers. If $\mathcal{E}_{i}(1)=\left(a_{i}, b_{i}\right)$ then

$$
T_{i}=E_{a_{i}, b_{i}}=\left\{\left(a_{i} t, b_{i} t\right)\left(\bmod \pi_{1}(A)\right) ; t \in \mathbb{C}\right\} \subset A .
$$

If the origin $\check{o}_{A} \notin T_{i}$, then for any point $\left(P_{i}, Q_{i}\right) \in T_{i}$ the elliptic curve $T_{i}=$ $E_{a_{i}, b_{i}}+\left(P_{i}, Q_{i}\right)$. In either case, all $v_{i}=\left(a_{i}, b_{i}\right)$ are eigenvectors of the linear part $g_{o}$ of $g=\tau_{p} g_{o} \in \operatorname{ker} \varphi$. We claim that there are at least three pairwise nonproportional $v_{i}$. Indeed, if all $v_{i}$ were parallel, then $T^{\operatorname{sing}}=\emptyset$, which contradicts $T_{i} \cap T^{\text {sing }} \neq \emptyset$ for $1 \leq i \leq h$ by Lemma 3 iii). Suppose that among $v_{1}, \ldots, v_{h}$ there are two non-parallel and all other $v_{i}$ are proportional to one of them. Then after an eventual permutation there is $1 \leq k \leq h-1$, such that $v_{1}, v_{k}$ are linearly 
independent, $v_{i}=\mu_{i} v_{1}$ for $\mu_{i} \in \mathbb{C}, 2 \leq i \leq k$ and $v_{i}=\mu_{i} v_{k+1}$ for $\mu_{i} \in$ $\mathbb{C}, k+2 \leq i \leq h$. Holzapfel has proved in [9] that any abelian ball quotient model $(A, T)$ is subject to $\sum_{i=1}^{h} \operatorname{card}\left(T_{i} \cap T^{\mathrm{sing}}\right)=4 \operatorname{card}\left(T^{\mathrm{sing}}\right)$. In the case under consideration

$$
\begin{gathered}
\operatorname{card}\left(T^{\text {sing }}\right)=\sum_{i=1}^{k} \sum_{j=k+1}^{h} \operatorname{card}\left(T_{i} \cap T_{j}\right) \\
\operatorname{card}\left(T_{i} \cap T^{\text {sing }}\right)=\sum_{j=k+1}^{h} \operatorname{card}\left(T_{i} \cap T_{j}\right) \quad \text { for } \quad 1 \leq i \leq k \quad \text { and } \\
\operatorname{card}\left(T_{j} \cap T^{\text {sing }}\right)=\sum_{i=1}^{k} \operatorname{card}\left(T_{i} \cap T_{j}\right) \quad \text { for } \quad k+1 \leq j \leq h .
\end{gathered}
$$

Therefore $\sum_{i=1}^{h} \operatorname{card}\left(T_{i} \cap T^{\text {sing }}\right)=2 \operatorname{card}\left(T^{\text {sing }}\right) \neq 4 \operatorname{card}\left(T^{\text {sing }}\right)$ and there are at least three pairwise non-proportional eigenvectors $v_{1}, v_{2}, v_{3}$ of $g_{o}$. Let $\lambda_{i}$ be the corresponding eigenvalues of $v_{i}$ and $v_{3}=\rho_{1} v_{1}+\rho_{2} v_{2}$ for some $\rho_{1}, \rho_{2} \in \mathbb{C}^{*}$. Then $\lambda_{3} v_{3}=g_{o}\left(v_{3}\right)=\rho_{1} \lambda_{1} v_{1}+\rho_{2} \lambda_{2} v_{2}$ implies that $\lambda_{1}=\lambda_{3}=\lambda_{2}$ and $g_{o}=\lambda_{o} I_{2}$ is a scalar matrix. On the other hand, $g\left(T_{i}\right)=g_{o}\left(T_{i}\right)+p=T_{i}$ for all $1 \leq$ $i \leq h$, so that $g_{o}$ permutes among themselves the parallel elliptic curves among $T_{1}, \ldots, T_{h}$. Since $T_{i}$ are finitely many, there is a natural number $m$, such that $g_{o}^{m} \in \operatorname{ker} \varphi$. Therefore, $\lambda_{o}^{m} \in \operatorname{End}\left(T_{i}\right)$ and $\lambda_{o}^{-m} \in \operatorname{End}\left(T_{i}\right)$ for all $1 \leq i \leq h$, due to $\left(g_{o}^{m}\right)^{-1}=g_{o}^{-m} \in \operatorname{ker} \varphi$. Recall that the units group $\operatorname{End}^{*}\left(T_{i}\right)=\mathbb{Z}^{*}=$ $\{ \pm 1\}$ for $T_{i}$ without a complex multiplication. If the elliptic curve $T_{i}$ has complex multiplication by an imaginary quadratic number field $\mathbb{Q}(\sqrt{-d}), d \in \mathbb{N}$, then $\operatorname{End}\left(T_{i}\right)$ is a subring of the integers ring $\mathcal{O}_{-d}$ of $\mathbb{Q}(\sqrt{-d})$. The units groups $\mathcal{O}_{-1}^{*}=\langle\mathrm{i}\rangle, \mathcal{O}_{-3}^{*}=\left\langle\mathrm{e}^{\frac{2 \pi i}{6}}\right\rangle$, and $\mathcal{O}_{-d}^{*}=\langle-1\rangle$ for all $d \neq 1,3$ are finite cyclic groups. As a subgroup of $\mathcal{O}_{-d}^{*}$, the units group $\operatorname{End}^{*}\left(T_{i}\right)$ is a finite cyclic group. Therefore $\lambda_{o}^{m} \in \operatorname{End}^{*}\left(T_{i}\right)$ and $g_{o}=\lambda_{o} I_{2}$ take finitely many values.

Concerning the translation part $\tau_{p}$ of $g \in \operatorname{ker} \varphi$, one can always move the origin $\check{o}_{A}$ of $A$ at one of the singular points of $T$. Due to the $G$-invariance of $T^{\text {sing }}$, there follows $g\left(\check{o}_{A}\right)=\tau_{p} g_{o}\left(\check{o}_{a}\right)=\tau_{p}\left(\check{o}_{A}\right)=p \in T^{\text {sing }}$. Therefore $p$ takes finitely many values and $\operatorname{ker} \varphi$ is finite.

ii) Since $\Gamma \subset \mathrm{SU}(2,1)$ is a torsion free lattice, any subgroup $H$ of

$$
G=\operatorname{Aut}\left(A^{\prime}, T^{\prime}\right) \subseteq \operatorname{Aut}\left(A^{\prime} \backslash T^{\prime}\right)=\operatorname{Aut}(\mathbb{B} / \Gamma)
$$


lifts to a subgroup $\Gamma_{H} \subset \operatorname{Aut}(\mathbb{B})=\mathrm{SU}(2,1)$, which normalizes $\Gamma$ and has quotient $\Gamma_{H} / \Gamma=H$. We claim that $\Gamma_{H}$ is discrete. Indeed, $\Gamma_{H}=\cup_{i=1}^{k} \gamma_{i} \Gamma$ is a finite disjoint union of cosets, relative to $\Gamma$. Suppose that $\Gamma_{H}$ is not discrete and there is a sequence $\left\{\nu_{n}\right\}_{n=1}^{\infty} \subset \Gamma_{H}$ with a limit point $\nu_{o} \in \gamma_{i_{o}} \Gamma$. Then pass to a subsequence $\left\{\nu_{m_{n}}\right\}_{n=1}^{\infty} \subset \gamma_{i_{o}} \Gamma$, converging to $\nu_{o}$. As a result $\left\{\gamma_{i_{o}}^{-1} \nu_{m_{n}}\right\}_{n=1}^{\infty} \subset \Gamma$ converges to $\gamma_{i_{o}}^{-1} \nu_{o} \in \Gamma$ and contradicts the discreteness of $\Gamma$. Thus, $\Gamma_{H} \supseteq \Gamma$ is discrete and, therefore, a ball lattice. Straightforwardly,

$$
A^{\prime} / H=\left[(\mathbb{B} / \Gamma) /\left(\Gamma_{H} / \Gamma\right)\right] \cup\left(T^{\prime} / H\right)=\left(\mathbb{B} / \Gamma_{H}\right) \cup\left(T^{\prime} / H\right)=\overline{\left(\mathbb{B} / \Gamma_{H}\right)}
$$

is the compactification of the ball quotient $\mathbb{B} / \Gamma_{H}$ by the divisor $T^{\prime} / H$. The $H$ Galois covers $\zeta_{H}: A \rightarrow A / H$ and $\zeta_{H}^{\prime}: A^{\prime} \rightarrow \overline{\left(\mathbb{B} / \Gamma_{H}\right)}$ fit in a commutative diagram

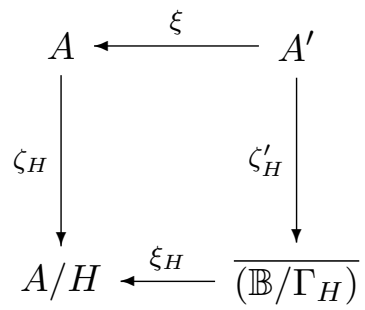

with the contraction $\xi_{H}$ of $L / H$ to $\xi(L) / H$.

Note that $X=A / H$ is smooth exactly when $H$ has no isolated fixed points on $A$. The blow-up $\xi: A^{\prime} \rightarrow A$ replaces an arbitrary $p_{j}=\xi\left(L_{j}\right)$ with stabilizer $\operatorname{Stab}_{H}\left(p_{j}\right)$ by a smooth rational curve $L_{j}$ with $\operatorname{Stab}_{H}(q)=\operatorname{Stab}_{H}\left(p_{j}\right)$ for all $q \in L_{j}$. Therefore the blow-up $\xi$ does not create isolated $H$-fixed points on $A^{\prime}$ and $A^{\prime} / H=\overline{\left(\mathbb{B} / \Gamma_{H}\right)}$ is a smooth compactification. Its open subset $\mathbb{B} / \Gamma_{H}$ is smooth.

\section{Explicit Constructions}

The present section applies Lemma 5 to a specific abelian ball quotient model over the Gauss numbers $\mathbb{Q}(\mathrm{i})$, in order to provide ball quotient compactifications, which are birational to a hyperelliptic, Enriques or a ruled surface with an elliptic base.

Theorem 6 (Holzapfel [9]) . Let us consider the elliptic curve $E_{-1}=\mathbb{C} /(\mathbb{Z}+\mathrm{i} \mathbb{Z})$ 
with complex multiplication by the Gauss numbers $\mathbb{Q}(\mathrm{i})$, its two-torsion points $Q_{0}=0(\bmod \mathbb{Z}+\mathrm{i} \mathbb{Z}), \quad Q_{1}=\frac{1}{2}(\bmod \mathbb{Z}+\mathrm{i} \mathbb{Z}), \quad Q_{2}=\mathrm{i} Q_{1}, \quad Q_{3}=Q_{1}+Q_{2}$ the abelian surface $A_{-1}=E_{-1} \times E_{-1}$, the points

$$
Q_{i j}=\left(Q_{i}, Q_{j}\right) \in A_{2-\text { tor }} \subset A_{-1}
$$

and the divisor $T_{-1}^{(6,8)}=\sum_{i=1}^{8} T_{i}$ with smooth elliptic irreducible components

$$
\begin{gathered}
T_{k}=E_{\mathrm{i}^{k}, 1} \quad \text { for } \quad 1 \leq k \leq 4 \\
T_{m+4}=Q_{m} \times E_{-1}, \quad T_{m+6}=E_{-1} \times Q_{m} \quad \text { for } \quad 1 \leq m \leq 2 .
\end{gathered}
$$

Then $\left(A_{-1}, T_{-1}^{(6,8)}\right)$ is an abelian model of an arithmetic ball quotient $\mathbb{B} / \Gamma_{-1}^{(6,8)}$, defined over $\mathbb{Q}(\mathrm{i})$.

\section{Corollary 7 (Holzapfel [9]) .}

i) In the notations from Theorem 6, the multiplications $I=\left(\begin{array}{ll}\mathrm{i} & 0 \\ 0 & 1\end{array}\right), J=$ $\left(\begin{array}{ll}1 & 0 \\ 0 & \mathrm{i}\end{array}\right)$ by $\mathrm{i} \in \mathbb{Z}[\mathrm{i}]=\operatorname{End}\left(E_{-1}\right)$ on the first, respectively, the second elliptic factor $E_{-1}$ of $A_{-1}$ are automorphisms of $\left(A_{-1}, T_{-1}^{(6,8)}\right)$.

ii) If $\Gamma_{K 3,-1}^{(6,8)}$ is the ball lattice, containing $\Gamma_{-1}^{(6,8)}$ as a normal subgroup with quotient $\Gamma_{K 3,-1}^{(6,8)} / \Gamma_{-1}^{(6,8)}=\left\langle-I_{2}=I^{2} J^{2}\right\rangle \subset \operatorname{Aut}\left(A_{-1}, T_{-1}^{(6,8)}\right)$, then the ball quotient $\mathbb{B} / \Gamma_{K 3,-1}^{(6,8)}$ is birational to the Kummer surface $X_{K 3}$ of $A_{-1}$.

iii) If $\Gamma_{\text {Rat,-1 }}^{(6,8)}$ is the ball lattice, containing $\Gamma_{-1}^{(6,8)}$ as a normal subgroup with quotient $\Gamma_{\mathrm{Rat},-1}^{(6,8)} / \Gamma_{-1}^{(6,8)}=\langle I, J\rangle \subseteq \operatorname{Aut}\left(A_{-1}, T_{-1}^{(6,8)}\right)$, then the ball quotient $\mathbb{B} / \Gamma_{\text {Rat },-1}^{(6,8)}$ is a rational surface.

The entire automorphism group $G_{-1}^{(6,8)}=\operatorname{Aut}\left(A_{-1}, T_{-1}^{(6,8)}\right)$ is described in the next lemma. 
Lemma 8. In the notations from Theorem 6, the group $G_{-1}^{(6,8)}=\operatorname{Aut}\left(A_{-1}, T_{-1}^{(6,8)}\right)$ is generated by $I=\left(\begin{array}{ll}\mathrm{i} & 0 \\ 0 & 1\end{array}\right), J=\left(\begin{array}{ll}1 & 0 \\ 0 & \mathrm{i}\end{array}\right)$, the transposition $\theta=\left(\begin{array}{ll}0 & 1 \\ 1 & 0\end{array}\right)$ of the elliptic factors $E_{-1}$ of $A_{-1}$ and the translation $\tau_{33}$ by $Q_{33}$. The aforementioned generators are subject to the relations

$$
\begin{gathered}
I^{4}=\mathrm{Id}, \quad J^{4}=\mathrm{Id}, \quad \theta^{2}=\mathrm{Id}, \quad \tau_{33}^{2}=\mathrm{Id}, \quad I J=J I \\
\theta I=J \theta, \quad \theta J=I \theta, \quad I \tau_{33}=\tau_{33} I, \quad J \tau_{33}=\tau_{33} J, \quad \theta \tau_{33}=\tau_{33} \theta .
\end{gathered}
$$

and $G_{-1}^{(6,8)}$ is of order 64 .

\section{Proof:}

Any $g \in G_{-1}^{(6,8)}$ leaves invariant

$$
\left(T_{-1}^{(6,8)}\right)^{\mathrm{sing}}=\sum_{1 \leq i<j \leq 8} T_{i} \cap T_{j}=\sum_{m=1}^{2} \sum_{n=1}^{2} Q_{m n}+Q_{00}+Q_{33} .
$$

Thus, $g\left(T_{i}\right)=T_{j}$ implies $s_{i}=\operatorname{card}\left(T_{i} \cap T^{\mathrm{sing}}\right)=\operatorname{card}\left(T_{j} \cap T^{\mathrm{sing}}\right)=s_{j}$, according to the bijectiveness of $g$. In the case under consideration, $s_{1}=s_{2}=$ $s_{3}=s_{4}=4$ and $s_{5}=s_{6}=s_{7}=s_{8}=2$, so that $G_{-1}^{(6,8)}$ permutes separately $T_{1}, \ldots, T_{4}$ and $T_{5}, \ldots, T_{8}$. In particular, the intersection $\cap_{i=1}^{4} T_{i}=\left\{Q_{00}, Q_{33}\right\}$ is $G_{-1}^{(6,8)}$-invariant and any $g=\tau_{(U, V)} g_{o} \in G_{-1}^{(6,8)}$ transforms the origin $\check{o}_{A_{-1}}=Q_{00}$ into $g\left(\check{o}_{A_{-1}}\right)=\left(U_{1}, U_{2}\right) \in\left\{Q_{00}, Q_{33}\right\}$. Straightforwardly, $\tau_{33}\left(T_{i}\right)=T_{i}$ for $1 \leq i \leq 4$ and $\tau_{33}\left(T_{m+2 n}\right)=T_{3-m+2 n}$ for $1 \leq m \leq 2,2 \leq n \leq 3$ imply that $\tau_{33} \in G_{-1}^{(6,8)}$. Therefore $G_{-1}^{(6,8)}$ is generated by $G_{-1}^{(6,8)} \cap \mathrm{GL}_{2}\left(\operatorname{End}\left(E_{-1}\right)\right)=$ $G_{-1}^{(6,8)} \cap \mathrm{GL}_{2}(\mathbb{Z}[\mathrm{i}])$ and $\tau_{33}$. Note that $\theta \in \operatorname{Aut}\left(A_{-1}\right)$ acts on $T_{-1}^{(6,8)}$ and induces the permutation $\left(T_{1}, T_{3}\right)\left(T_{5}, T_{7}\right)\left(T_{6}, T_{8}\right)$ of its irreducible components. Therefore $\theta \in G_{-1}^{(6,8)}$ and $\langle I, J, \theta\rangle$ is a subgroup of $G_{-1}^{(6,8)} \cap \mathrm{GL}_{2}(\mathbb{Z}[\mathrm{i}])$. On the other hand, any $g=\left(\begin{array}{cc}\alpha & \beta \\ \gamma & \delta\end{array}\right) \in G_{-1}^{(6,8)} \cap \mathrm{GL}_{2}(\mathbb{Z}[\mathrm{i}])$ acts on $T_{5}, \ldots, T_{8}$ and, therefore, on the set $\left\{\widetilde{T_{5}}=\widetilde{T_{6}}=0 \times \mathbb{C}, \widetilde{T_{7}}=\widetilde{T_{8}}=\mathbb{C} \times 0\right\}$ of the corresponding universal covers. If $g(0 \times \mathbb{C})=0 \times \mathbb{C}, g(\mathbb{C} \times 0)=\mathbb{C} \times 0$ then $\beta=\gamma=0$, so that $\alpha, \delta \in \operatorname{End}\left(E_{-1}\right)=\mathbb{Z}[\mathrm{i}]$ and $\operatorname{det}(g)=\alpha \delta \in \operatorname{End}^{*}\left(E_{-1}\right)=\langle\mathrm{i}\rangle=\mathbb{C}_{4}$ imply $g=I^{k} J^{l}$ for some $0 \leq k, l \leq 3$. Similarly, for $g(0 \times \mathbb{C})=\mathbb{C} \times 0, g(\mathbb{C} \times 0)=0 \times \mathbb{C}$ one has $\alpha=\delta=0$, whereas $\beta, \gamma \in \mathbb{Z}[\mathrm{i}], \beta \gamma \in \mathbb{Z}[\mathrm{i}]^{*}=\langle\mathrm{i}\rangle$ and $g=I^{k} J^{l} \theta$ for some $0 \leq k, l \leq 3$. Consequently, $G_{-1}^{(6,8)} \cap \mathrm{GL}_{2}(\mathbb{Z}[\mathrm{i}])=\langle I, J, \theta\rangle$ and $G_{-1}^{(6,8)}=$ $\left\langle I, J, \theta, \tau_{33}\right\rangle$. The announced relations among $\tau_{33}, I, J, \theta$ imply that

$$
G_{-1}^{(6,8)}=\left\{\tau_{33}^{n} I^{k} J^{l} \theta^{m} ; 0 \leq k, l \leq 3, \quad 0 \leq m, n \leq 1\right\}
$$


is of order 64 .

Theorem 9. In the notations from Lemma 5, Theorem 6 and Lemma 8, let us consider the subgroups $H_{H E}=\left\langle\tau_{33} J^{2}\right\rangle, H_{\mathrm{Enr}}=\left\langle-I_{2}, \tau_{33} I^{2}\right\rangle, H_{\mathrm{Rul}}=\left\langle J^{2}\right\rangle$ of $G_{-1}^{(6,8)}=\operatorname{Aut}\left(A_{-1}, T_{-1}^{(6,8)}\right)$, their liftings $\Gamma_{H E,-1}^{(6,8)}, \Gamma_{\mathrm{Enr},-1}^{(6,8)}, \Gamma_{\mathrm{Rul},-1}^{(6,8)}$ to ball lattices and the blow-up $A_{\widehat{2-\text { tor }}}$ of $A_{-1}$ at the two-torsion points $A_{2-\text { tor. Then }}$

i) $\mathbb{B} / \Gamma_{H E,-1}^{(6,8)}$ is a smooth ball quotient, birational to the smooth hyperelliptic surface $A_{-1} / H_{H E}$

ii) $\mathbb{B} / \Gamma_{\mathrm{Enr},-1}^{(6,8)}$ is a ball quotient with one double point $\operatorname{Orb}_{H_{\mathrm{Enr}}}\left(Q_{03}\right)$, which is birational to the smooth Enriques surface $A_{\widehat{2-\text { tor }}} / H_{\mathrm{Enr}}$

iii) $\mathbb{B} / \Gamma_{\mathrm{Rul},-1}^{(6,8)}$ is a smooth ball quotient, birational to the smooth trivial ruled surface $A_{-1} / H_{\mathrm{Rul}}=E_{-1} \times \mathbb{P}^{1}$ with an elliptic base $E_{-1}$.

Proof: i) Recall that the $\mathbb{Z}$-module $\pi_{1}\left(E_{-1}\right)=\mathbb{Z}+\mathrm{i} \mathbb{Z}=\mathbb{Z}+(1+\mathrm{i}) \mathbb{Z}$ is generated by $1,1+\mathrm{i}$ and $Q_{3}=\frac{1+\mathrm{i}}{2}\left(\bmod \pi_{1}\left(E_{-1}\right)\right)$. The translation $\tau_{Q_{3}}: E_{-1} \rightarrow E_{-1}$ is of order 2 , as well as the morphism

$$
\begin{gathered}
\tau_{Q_{3}}(-1): E_{-1} \longrightarrow E_{-1} \\
\tau_{Q_{3}}(-1)(P)=-P+Q_{3}
\end{gathered}
$$

with four fixed points

$$
\frac{1}{2} Q_{3}+\left(E_{-1}\right)_{2-\text { tor }}=\frac{1}{2} Q_{3}+\left\{Q_{i} ; 0 \leq i \leq 3\right\} .
$$

According to [5], the quotient $A_{-1} / H_{H E}$ by the cyclic group

$$
H_{H E}=\left\langle\tau_{Q_{3}} \times \tau_{Q_{3}}(-1)\right\rangle
$$

of order 2 is a smooth hyperelliptic surface. Lemma 5 ii) implies that $\mathbb{B} / \Gamma_{H E,-1}^{(6,8)}$ is a smooth ball quotient, birational to $A_{-1} / H_{H E}$.

ii) The quotient $X_{K 3}=A_{\widehat{2-\text { tor }}} /\left\langle-I_{2}\right\rangle$ is a smooth $\mathrm{K} 3$ surface, called the Kummer surface of $A_{-1}$. We claim that the involution $\tau_{33} I^{2}$ acts on $A_{\widehat{2-\text { tor }}}$ and determines an unramified double cover

$$
\zeta: X_{K 3}=A_{\widehat{2-\text { tor }}} /\left\langle-I_{2}\right\rangle \rightarrow A_{\widehat{2-\text { tor }}} /\left\langle-I_{2}, \tau_{33} I^{2}\right\rangle=A_{\widehat{2-\text { tor }}} / H_{\mathrm{Enr}} .
$$


More precisely, $\tau_{33} I^{2}=\tau_{Q_{3}}(-1) \times \tau_{Q_{3}}$ leaves invariant the two-torsion points $A_{2-\text { tor }}=\left\{Q_{i j} ; 0 \leq i, j \leq 3\right\}$ and any choice of an automorphism of $\mathbb{P}^{1}$ extends $\tau_{33} I^{2}$ to an automorphism of $A_{\widehat{2-\text { tor }}}$. Note that $\tau_{33} I^{2}\left(-I_{2}\right)=\left(-I_{2}\right) \tau_{33} I^{2}$, so that $\tau_{33} I^{2}$ normalizes $\left\langle-I_{2}\right\rangle$ and there is a well defined quotient group $H_{\text {Enr }} /\left\langle-I_{2}\right\rangle=$ $\left\langle\tau_{33} I^{2}\right\rangle$ of order 2 . That allows to define $\zeta: X_{K 3} \rightarrow A_{\widehat{2-\text { tor }}} / H_{\text {Enr as an }}$ $H_{\mathrm{Enr}} /\left\langle-I_{2}\right\rangle$-Galois cover. We claim that $\tau_{33} I^{2}$ is a fixed point free involution on $X_{K 3}$, in order to conclude that $A_{\widehat{2-\text { tor }}} / H_{\mathrm{Enr}}$ is a smooth Enriques surface. More precisely, the fixed points of $\tau_{33} I^{2}$ on the set $X_{K 3}$ of the $\left\langle-I_{2}\right\rangle$-orbits on $A_{\widehat{2-\text { tor }}}$ lift to $\varepsilon$-fixed points of $\tau_{33} I^{2}$ on $A_{\widehat{2-\text { tor }}}$ for $\varepsilon= \pm 1$. The $\varepsilon$-fixed points $(P, Q) \in A_{-1}$ are subject to

$$
\begin{aligned}
-P+Q_{3} & =\varepsilon P \\
Q+Q_{3} & =\varepsilon Q .
\end{aligned}
$$

For $\varepsilon=1$ the equality $Q+Q_{3}=Q$ has no solution $Q \in E_{-1}$, while for $\varepsilon=-1$ the equation $-P+Q_{3}=-P$ on $P \in E_{-1}$ is inconsistent. Therefore $\tau_{33} I^{2}$ has no $\varepsilon$-fixed points on $A_{-1}$. By the very definition of the $\tau_{33} I^{2}$-action on $A_{\widehat{2-\text { tor }}}$, there are no $\varepsilon$-fixed points for $\tau_{33} I^{2}$ on $A_{\widehat{2-\text { tor }}}$ and $\tau_{33} I^{2}: X_{K 3} \rightarrow X_{K 3}$ is a fixed point free involution. As a result, $A_{\widehat{2-\text { tor }}} / H_{\mathrm{Enr}}$ is a smooth Enriques surface.

Recall that the exceptional divisor $\xi_{2-\text { tor }}^{-1}\left(A_{2-\text { tor }}\right)$ of the blow-up

$$
\xi_{2-\text { tor }}: A_{\widehat{2-\text { tor }}}^{\rightarrow} A_{-1}
$$

of $A_{-1}$ at $A_{2-\text { tor }}$ is $H_{\mathrm{Enr}}$-invariant, so that $\xi_{2-\text { tor }}$ descends to the contraction $\overline{\xi_{2-\text { tor }}}: A_{\widehat{2-\text { tor }}} / H_{\text {Enr }} \rightarrow A_{-1} / H_{\text {Enr }}$ of $\xi_{2-\text { tor }}^{-1}\left(A_{2-\text { tor }}\right) / H_{\text {Enr }}$ to $A_{2-\text { tor }} / H_{\text {Enr }}$. In particular, the smooth Enriques surface $A_{\widehat{2-\text { tor }}} / H_{\text {Enr }}$ is birational to $A_{-1} / H_{\text {Enr }}$. The singular locus $\left(A_{-1} / H_{\text {Enr }}\right)^{\text {sing }} \subseteq\left(A_{2-\text { tor }} / H_{\text {Enr }}\right)$, according to the smoothness of $A_{\widehat{2-\text { tor }}} / H_{\mathrm{Enr}}$. On the other hand, $\tau_{33} I^{2}$ has no fixed points on $A_{2-\text { tor }}$, so that $A_{2-\text { tor }} / H_{\text {Enr }}$ consists of eight double points

$$
\operatorname{Orb}_{H_{\text {Enr }}}\left(Q_{i j}\right)=\operatorname{Orb}_{H_{\text {Enr }}}\left(Q_{3-i, 3-j}\right), \quad 0 \leq i, j \leq 3
$$

and $\left(A_{-1} / H_{\text {Enr }}\right)^{\text {sing }}=A_{2-\text { tor }} / H_{\text {Enr }}$. Note that

$$
\left(T_{-1}^{(6,8)}\right)^{\operatorname{sing}}=\left\{\operatorname{Orb}_{H_{\mathrm{Enr}}}\left(Q_{00}\right), \operatorname{Orb}_{H_{\mathrm{Enr}}}\left(Q_{11}\right), \operatorname{Orb}_{H_{\mathrm{Enr}}}\left(Q_{12}\right)\right\}
$$

is contained in $\left(A_{-1} / H_{\mathrm{Enr}}\right)^{\mathrm{sing}}$ and the birational morphism

$$
\xi_{H_{\mathrm{Enr}}}:\left(\overline{\mathbb{B} / \Gamma_{\mathrm{Enr},-1}^{(6,8)}}\right) \rightarrow A_{-1} / H_{\mathrm{Enr}}
$$


resolves $\left(T_{-1}^{(6,8)}\right)^{\text {sing }}$ by smooth rational curves of self-intersection $(-2)$. Therefore $\left(\overline{\mathbb{B} / \Gamma_{\text {Enr,-1 }}^{(6,8)}}\right)^{\text {sing }}$ consists of the following five double points:

$\operatorname{Orb}_{H_{\text {Enr }}}\left(Q_{01}\right), \operatorname{Orb}_{H_{\text {Enr }}}\left(Q_{10}\right), \operatorname{Orb}_{H_{\text {Enr }}}\left(Q_{02}\right), \operatorname{Orb}_{H_{\text {Enr }}}\left(Q_{20}\right), \operatorname{Orb}_{H_{\text {Enr }}}\left(Q_{03}\right)$.

Since

$$
\begin{aligned}
& \operatorname{Orb}_{H_{\text {Enr }}}\left(Q_{0, m}\right) \in\left[T_{m+6} \backslash\left(T_{-1}^{(6,8)}\right)^{\mathrm{sing}}\right] / H_{\mathrm{Enr}}=\left(T_{m+6}^{\prime} \backslash L\right) / H_{\mathrm{Enr}} \\
& \operatorname{Orb}_{H_{\mathrm{Enr}}}\left(Q_{m, 0}\right) \in\left[T_{m+4} \backslash\left(T_{-1}^{(6,8)}\right)^{\mathrm{sing}}\right] / H_{\mathrm{Enr}}=\left(T_{m+4}^{\prime} \backslash L\right) / H_{\mathrm{Enr}}
\end{aligned}
$$

for all $1 \leq m \leq 2$ belong to the compactifying divisor $T^{\prime} / H_{\mathrm{Enr}}$, the ball quotient $\mathbb{B} / \Gamma_{\mathrm{Enr},-1}^{(6,8)}$ has only one singular point

$$
\left(\mathbb{B} / \Gamma_{\text {Enr,-1 }}^{(6,8)}\right)^{\text {sing }}=\left\{\operatorname{Orb}_{H_{\text {Enr }}}\left(Q_{0,3}\right)\right\}
$$

iii) The quotient $X=A_{-1} / H_{\mathrm{Rul}}=E_{-1} \times\left[E_{-1} /\langle(-1)\rangle\right]$ of $A_{-1}$ by the reflection $J^{2}=1 \times(-1)$ is a smooth surface, birational to the smooth ball quotient $\mathbb{B} / \Gamma_{\mathrm{Rul},-1}^{(6,8)}$. It is well known that $C=E_{-1} /\langle-1\rangle$ is a smooth projective curve. More precisely, if

$$
\mathfrak{p}(t)=\frac{1}{t^{2}}+\sum_{\lambda \in(\mathbb{Z}+\mathrm{i} \mathbb{Z}) \backslash\{0\}}\left[\frac{1}{(t-\lambda)^{2}}-\frac{1}{\lambda^{2}}\right]
$$

is the Weierstrass $\mathfrak{p}$-function, associated with the lattice $\mathbb{Z}+\mathrm{i} \mathbb{Z}=\pi_{1}\left(E_{-1}\right)$, then the map

$$
\begin{gathered}
\psi: E_{-1} \backslash\left\{\check{o}_{E_{-1}}\right\} \longrightarrow \mathbb{P}^{2} \\
\psi(t+(\mathbb{Z}+\mathrm{i} \mathbb{Z}))=\left[1: \mathfrak{p}(t+(\mathbb{Z}+\mathrm{i} \mathbb{Z})): \mathfrak{p}^{\prime}(t+(\mathbb{Z}+\mathrm{i} \mathbb{Z}))\right]=\left[1: \mathfrak{p}(t): \mathfrak{p}^{\prime}(t)\right]
\end{gathered}
$$

extends by $\psi\left(\check{o}_{E_{-1}}\right)=[0: 0: 1]=p_{\infty}$ to a projective embedding of $E_{-1}$. The image

$$
\psi\left(E_{-1}\right)=\left\{[z: x: y] \in \mathbb{P}^{2} ; z y^{2}=\left(x-\mathfrak{p}\left(Q_{1}\right)\right)\left(x-\mathfrak{p}\left(Q_{2}\right)\right)\left(x-\mathfrak{p}\left(Q_{3}\right)\right)\right\}
$$

is a cubic hypersurface in $\mathbb{P}^{2}$. As far as $\mathfrak{p}(t)$ is even and $\mathfrak{p}^{\prime}(t)$ is an odd function of $t$, the multiplication $\mu_{-1}$ by -1 on $E_{-1}$ acts on $\psi\left(E_{-1}\right)$ by the rule

$$
\mu_{-1}([z: x: y])=[z: x:-y] \text {. }
$$


The fixed points of this action are $p_{\infty}$ and $\mathfrak{p}\left(Q_{i}\right)$ for $1 \leq i \leq 3$. The fibres of the projection

$$
\begin{gathered}
\Pi: \psi\left(E_{-1}\right) \backslash\left\{p_{\infty}\right\} \longrightarrow \mathbb{P}^{1} \backslash\left\{q_{\infty}=[0: 1]\right\} \\
\Pi([z: x: y])=[z: x]
\end{gathered}
$$

are exactly the $\mu_{-1}$-orbits on $\psi\left(E_{-1}\right) \backslash\left\{p_{\infty}\right\}$, so that its image

$$
\mathbb{P}^{1} \backslash\left\{q_{\infty}\right\}=\Pi\left(\psi\left(E_{-1}\right) \backslash\left\{p_{\infty}\right\}\right)=\left(\psi\left(E_{-1}\right) \backslash\left\{p_{\infty}\right\}\right) /\left\langle\mu_{-1}\right\rangle
$$

is the corresponding Galois quotient by the cyclic group $\left\langle\mu_{-1}\right\rangle$ of order 2 . Thus, $\psi\left(E_{-1}\right) /\left\langle\mu_{-1}\right\rangle=\left(\psi\left(E_{-1}\right) \backslash\left\{p_{\infty}\right\}\right) /\left\langle\mu_{-1}\right\rangle \cup\left\{p_{\infty}\right\}=\left(\mathbb{P}^{1} \backslash\left\{q_{\infty}\right\}\right) \cup\left\{p_{\infty}\right\}=\mathbb{P}^{1}$.

\section{References}

[1] Dzambic A., Arithmetic of a Fake Projective Plane and Related Elliptic Surfaces, arXiv : 0803.0645.

[2] Hirzebruch F. Chern Numbers of Algebraic Surfaces - An Example, Math. Ann. 266 (1984) 351-356.

[3] Holzapfel R.-P., Chern Numbers of Algebraic Surfaces - Hirzebruch's Examples Are Picard Modular Surfaces, Math. Nach. 126 (1986) 255-273.

[4] Holzapfel R.-P., Ball and Surface Arithmetic, Vieweg, Braunschweig 1998.

[5] Griffiths Ph. and Harris J., Principles of Algebraic Geometry, Wiley, New York 1978.

[6] Holzapfel R.-P. (with Appendices by A. Pineiro and N. Vladov), PicardEistein Metrics and Class Fields Connected with Apolonius Cycle, HU Preprint 98-15 (1998).

[7] Holzapfel R.-P., Jacobi Theta Embedding of a Hyperbolic 4-space with Cusps, In: Geometry, Integrability and Quantization III, I. Mladenov and G. Naber (Eds), Coral Press, Sofia 2002, pp 11-63.

[8] Holzapfel R.-P. and Vladov N., Quadric-Line Configurations Degenerating Plane Picard Einstein Metrics I \& II, Sitzungsber d. Berliner Math. Ges., Berlin 2003, pp 79-142.

[9] Holzapfel R.-P., Complex Hyperbolic Surfaces of Abelian Type, Serdica Math. J. 30 (2004) 207-238. 
[10] Ishida M., An Elliptic Surface Covered by Mumford's Fake Projective Plane, Tohoku Math. J. 40 (1988) 367-398.

[11] Keum J., A Fake Projective Plane with an Order 7 Automorphism, Topology 45 (2006) 919-927.

[12] Keum J., Quotients of Fake Projective Planes, Geom. Topol. 12 (2008) 2497-2515.

\author{
Azniv Kasparian \\ Department of Mathematics and Informatics \\ Kliment Ohridski University of Sofia \\ 1164 Sofia, BULGARIA \\ E-mail address: kasparia@fmi .uni-sofia.bg \\ Boris Kotzev \\ Department of Mathematics and Informatics \\ Kliment Ohridski University of Sofia \\ 1164 Sofia, BULGARIA \\ E-mail address: bkotzev@fmi .uni-sofia.bg
}

